\title{
Management of orbital cellulitis: a retrospective study
}

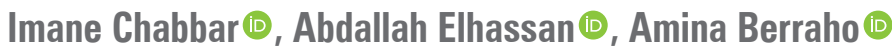 \\ Ophthalmology B Department, Ibn Sina University Hospital, Rabat, Morocco
}

\begin{abstract}
BACKGROUND: Orbital cellulitis is an infectious involvement of the tissues posterior to the orbital septum causing severe local and general complications.

MATERIAL AND METHODS: The aim of our work was to study the clinical, paraclinical and therapeutic characteristics of orbital cellulitis through a retrospective study of 89 cases collected between 2015 and 2019. The diagnosis of orbital cellulitis was based on clinical and imaging elements.

RESULTS: The average age was 17.5 years. Exophthalmos was noted in 33\% of cases, ophthalmoplegia in $18 \%$ of cases, diplopia in $4.5 \%$ of cases and ocular hypertonia in $11 \%$ of cases. Orbital CT scan allowed the diagnosis of subperiosteal abscess in $20 \%$ of cases and orbital abscess in $10 \%$ of cases. Sinusitis was the main infectious origin in children, while dacryocystitis predominated in adults. All patients received parenteral antibiotherapy combined with corticotherapy after 48 hours. Complications occurred in 10 cases, dominated by exposure keratitis, panophthalmitis and optic atrophy with permanent blindness.

CONCLUSIONS: We emphasize the importance of rapid diagnosis and urgent treatment of orbital cellulitis.
\end{abstract}

KEY WORDS: orbital cellulitis; management; complications; prognosis

Ophthalmol J 2020; Vol. 5, 87-92

\section{INTRODUCTION}

Orbital cellulitis is the most common primary orbital pathology. It is an acute orbital infection usually of bacterial origin. The diagnosis of orbital cellulitis is mainly clinical, confirmed by orbital CT scan. Chandler's classification (Tab. 1) allows to group orbital cellulitis into 2 main categories: preseptal cellulitis which describes the infection located before the orbital septum and "real" orbital cellulitis or postseptal cellulitis which involves the tissues posterior to the orbital septum and which is studied in this work.

Orbital cellulitis is a serious pathology causing severe local and general complications. Its unpredictable progression requires early diagnosis and appropriate and effective treatment. The difficulty of management is mainly seen at the stage of complications.
The aim of our work was to study the clinical, paraclinical, therapeutic and evolutive characteristics of orbital cellulitis through 5-years' experience.

\begin{tabular}{|l|l|}
\hline \multicolumn{2}{|l|}{ Table 1. Chandler's classification of orbital cellulitis } \\
\hline Stage & Description \\
\hline I & $\begin{array}{l}\text { Pre-septal cellulitis: inflammatory oedema of the } \\
\text { eyelids }\end{array}$ \\
\hline II & $\begin{array}{l}\text { True orbital cellulitis: diffuse oedema of the orbital } \\
\text { contents, posterior of the septum }\end{array}$ \\
\hline III & $\begin{array}{l}\text { Subperiosteal abscess: collection of purulent material } \\
\text { between the periorbit and the orbital wall (usually } \\
\text { medial or superolateral) }\end{array}$ \\
\hline IV & $\begin{array}{l}\text { Orbital abscess: abscess collection within the orbital } \\
\text { tissues }\end{array}$ \\
\hline V & Cavernous sinus thrombosis \\
\hline
\end{tabular}




\section{MATERIAL AND METHODS}

We conducted a retrospective study of patients presenting orbital cellulitis, hospitalized in Ophthalmology B Department at Ibn-Sina University Hospital in Rabat-Morocco, for a period of five years (From January 2015 to December 2019).

For each patient, we noted demographic parameters (age, sex, origin), the patient's medical history, the consultation delay, functional signs on admission and initial visual acuity. The slit-lamp examination specified: the presence of eyelids edema, chemosis, fistulization, exophthalmos, lagophthalmos, ophthalmoplegia, the anterior segment evaluation (degree of corneal transparency, anterior chamber inflammation, intraocular pressure measure) and finally the fundus eye examination. All patients underwent an urgent orbital CT scan. The diagnosis of orbital cellulitis was retained on clinical and imaging arguments.

Blood tests (complete blood count, electrolyte panel, C-reactive protein test) and microbiological examinations of purulent material were carried out during hospitalization.

We noted the medical and/or surgical treatment received by each patient, the modality of administration, the evolution after treatment and the complications.

\section{RESULTS AGE, SEX}

In our case series, 89 patients were treated for orbital cellulitis. There were 45\% males and 55\% females. The average age was 17.5 years with a range of 24 months to 68 years. The age group $<18$ years represented $38 \%$.

\section{IMIMUNE STATUS}

All patients were immunocompetent, except one patient who received chemotherapy for non-Hodgkin's lymphoma and 2 patients under long-term systemic corticotherapy for chronic rheumatic disease. Unbalanced diabetes was associated with 12 cases.

\section{VISUAL ACUITY ON ADMISSION}

Initial visual acuity was $>5 / 10$ in $22 \%$ of patients, while 5 patients had a negative light perception on admission (Fig. 1). However, visual acuity was not determined in $33 \%$ of cases.

\section{CLINICAL SIGNS}

On ophthalmological examination, the clinical signs were (Tab. 2): inflammatory edema limiting

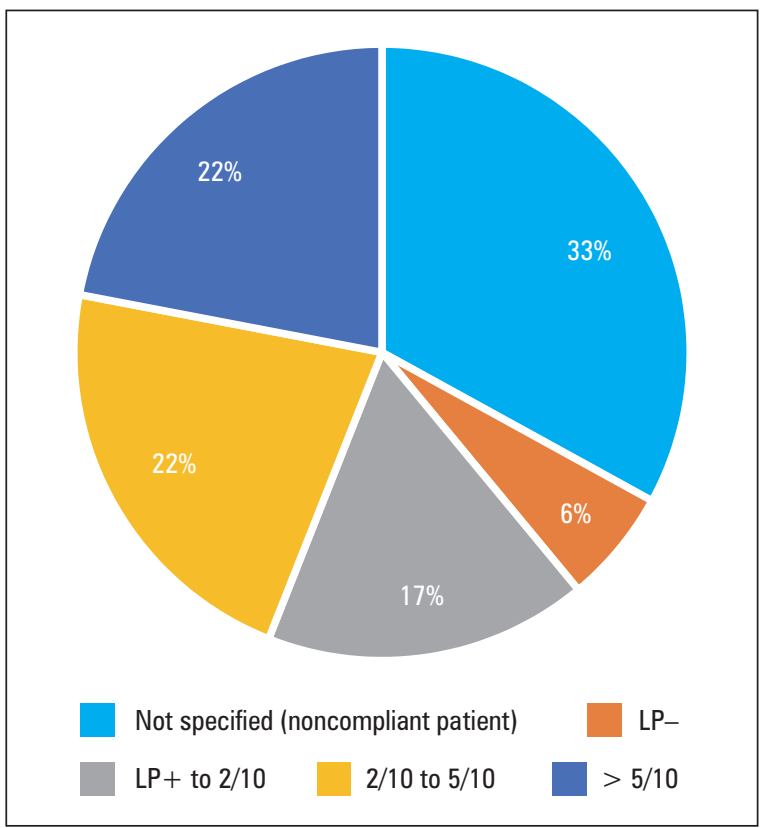

FIGURE 1. Distribution of cases according to initial visual acuity

Table 2. Table showing different clinical signs of orbital cellulitis

\begin{tabular}{|l|c|}
\hline Clinical signs & Rate \\
\hline Fever/reduced general condition & $11.2 \%$ \\
\hline Periorbital pain & $95.5 \%$ \\
\hline Eyelid edema/chemosis & $100 \%$ \\
\hline Exophthalmos & $33 \%$ \\
\hline Ophthalmoplegia & $18 \%$ \\
\hline Ocular hypertonia & $4.5 \%$ \\
\hline Diplopia & $11 \%$ \\
\hline
\end{tabular}

eyelids opening with chemosis in all patients, exophthalmos in $33 \%$ of cases (Fig. 2), ophthalmoplegia in $18 \%$ of cases, diplopia in $4.5 \%$ of cases and ocular hypertonia in $11 \%$ of cases. Fever with the reduced general condition was particularly objectified in young children.

\section{ORBITAL CT SCAN SIGNS}

Orbital CT scan allowed the diagnosis of orbital cellulitis in all patients, complicated by a subperiosteal abscess in $20 \%$ of cases (Fig. 3) and an orbital abscess in $10 \%$ of cases (Fig. 4). It also allowed the exophthalmos measurement (Fig. 5) and the diagnosis of related neighbouring infections.

\section{INITIAL INFECTIOUS ORIGIN}

Sinusitis was the main infectious origin in children (32 cases) while dacryocystitis was predomi- 


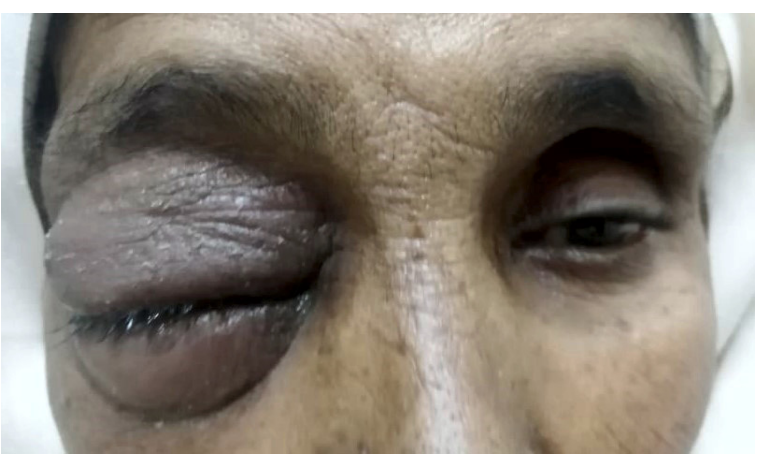

FIGURE 2. Orbital cellulitis in a 62-year-old woman: Photo showing eyelid edema with manifest exophthalmos
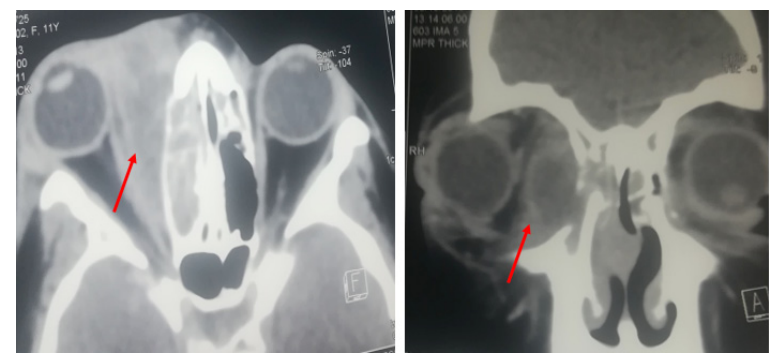

FIGURE 3. Orbital CT scan showing orbital cellulitis with subperiosteal abscess (arrow) in the right orbit complicating pansinusitis

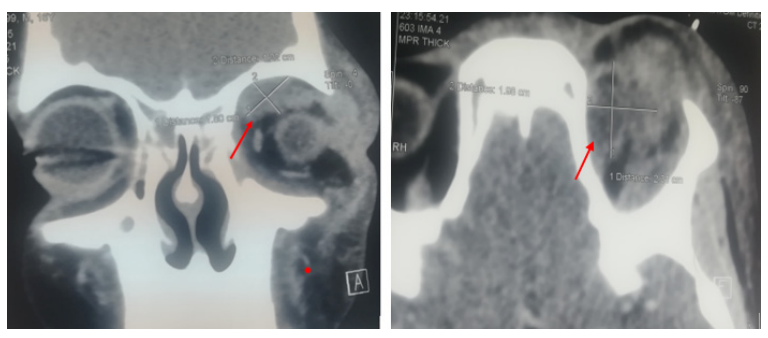

FIGURE 4. Orbital CT scan showing left orbital cellulitis complicated by an orbital abscess (arrow)

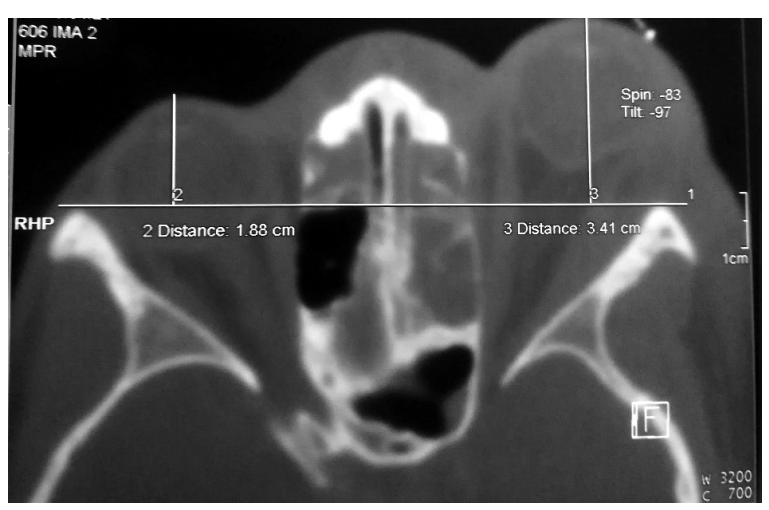

FIGURE 5, Orbital CT scan showing left orbital cellulitis with exophthalmos grade III complicating an acute ethmoiditis

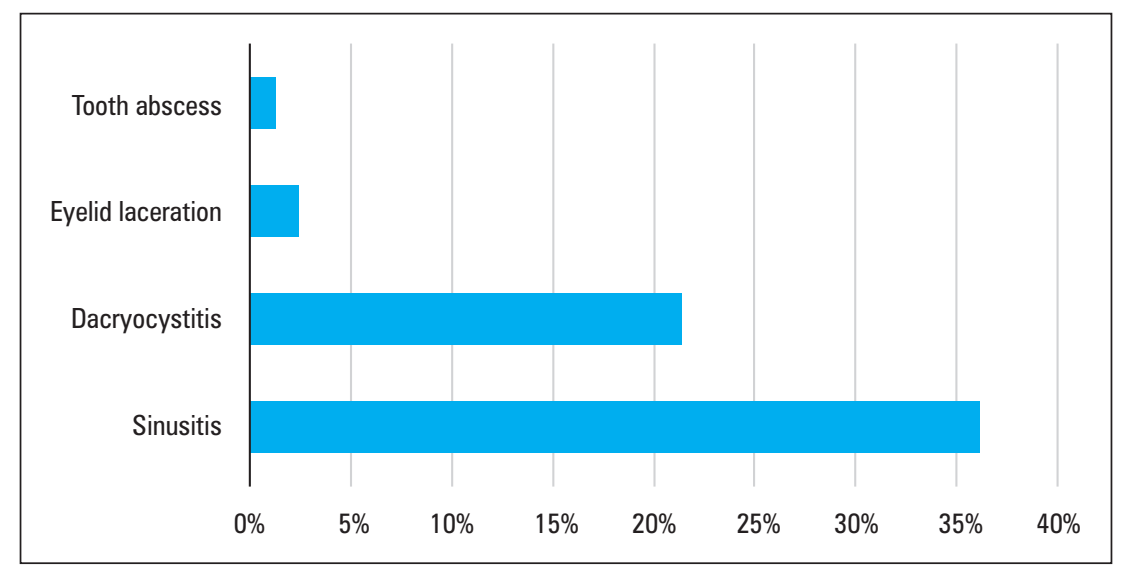

FIGURE 6. Distribution of cases according to initial infectious origin

nant in adults (19 cases) (Fig. 6). Two cases of eyelid laceration with a retained foreign body (Fig. 7) and one case of dental abscess were found.

\section{BACTERIAL IDENTIFICATION}

Microbiological examinations of purulent material taken either at the fistulization site or by needle puncture allowed the identification of bacteria in only $13 \%$ of cases. Isolated germes were respectively (Fig. 8): streptococcus pneumoniae, staphylococcus aureus, haemophilus influenzae and streptococcus mitis.

\section{TREATMENT}

Medical treatment required urgent hospitalization and initiation of parenteral antibiotherapy based on amoxicillin/clavulanic acid or ceftriaxone 


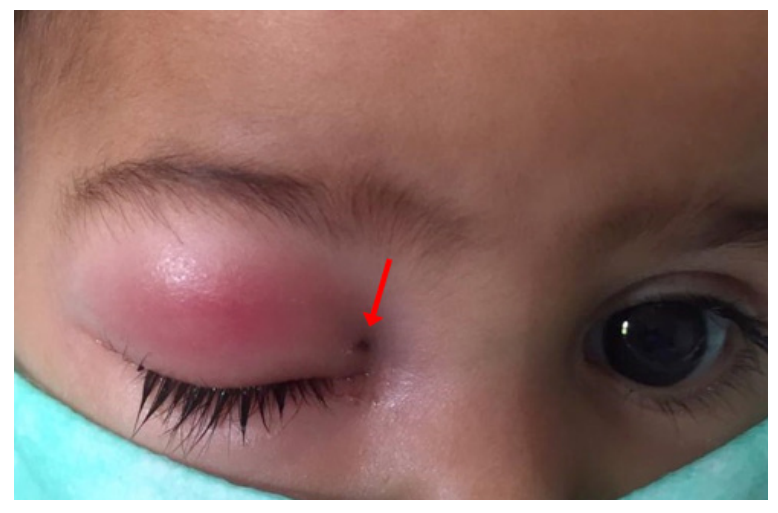

FIGURE 7. Orbital cellulitis in a 24-month old infant; photo showing a punctiform wound with a vegetable foreign body (arrow) at the medial canthus, with inflammatory edema limiting the eyelid opening

associated with aminoglycoside. Metronidazole was added if there was any doubt about the presence of anaerobic germs. Systemic corticotherapy was considered after 48 hours of effective antibiotherapy. Surgical treatment consisted of prefistulated collections drainage or needle puncture when the collection was deep.

\section{COMPLICATIONS}

The evolution after treatment was favorable in the majority of cases. Complications were encountered in $11.2 \%$ of cases, such as exposure keratitis, panophthalmitis, optic atrophy with permanent blindness and retinal detachment (Tab. 3).
Table 3. Distribution of cases according to orbital cellulitis complications

\begin{tabular}{|l|c|}
\hline Complications & Number of cases \\
\hline Exposure keratitis & 5 \\
\hline Optic atrophy & 2 \\
\hline Panophthalmitis & 2 \\
\hline Retinal detachment & 1 \\
\hline
\end{tabular}

\section{DISCUSSION}

Orbital cellulitis is an inflammatory process of infectious origin involving the tissues posterior to the orbital septum. The morbidity and mortality associated with orbital cellulitis have greatly decreased thanks to diagnostic and therapeutic advances. However, prompt diagnosis and urgent treatment remain crucial.

Although that can occur at any age, orbital cellulitis is more common in the paediatric population [1]. In our study, the mean age was 17.5 years with a range of 24 months to 65 years and $38 \%$ of cases under 18 years. Wane et al. [2] reported an average age of 18 years with a range of 7 months to 50 years. Kaimbo et al. [3] found an average age of 68 years with a significant proportion of patients for more than forty years. In the various published paediatric series [4-6], the age varied between 6 months and 14 years with an average of 5 years. Orbital cellulitis mainly affects young male patients [1]. However, we found a slight female predominance, also objectified in Aidan et al. study [7].

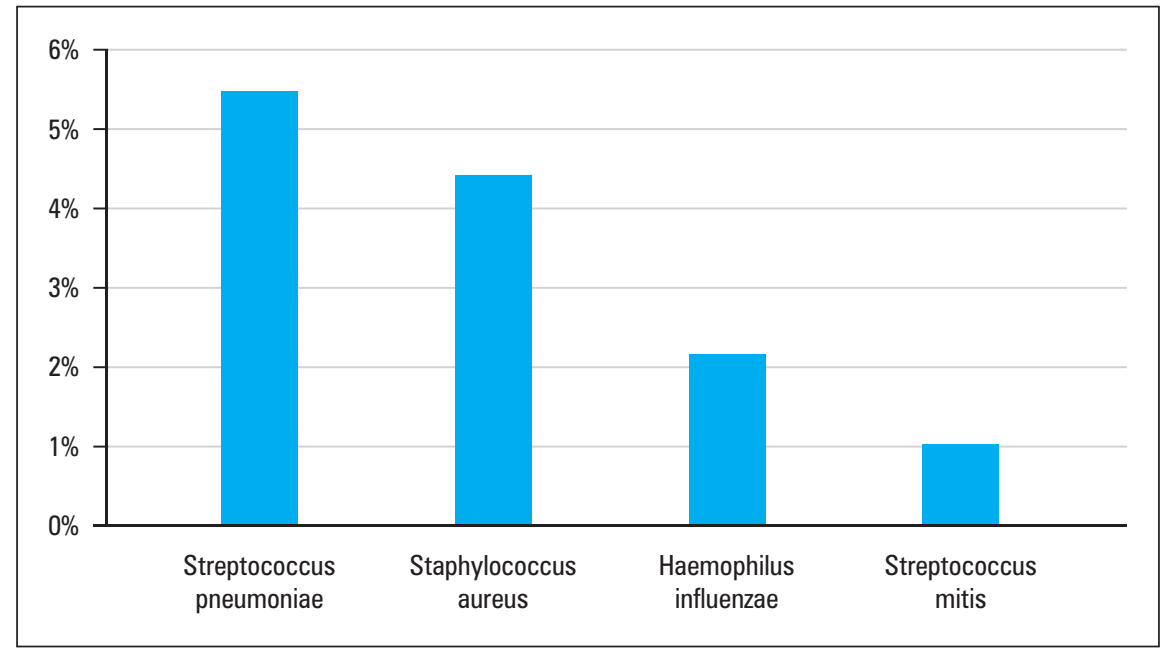

FIGURE 8. Bacterial identification in orbital cellulitis cases 
The clinical signs depend on the location of the infection objectified by Chandler's anatomo-clinical classification [8]. The presence of inflammatory eyelids edema with chemosis is characteristic. They were constantly present in all our cases. An inadequate initial treatment, prescribed on an outpatient basis or by self-medication, was the cause of a delayed consultation and a source of complications. The patients presented with a rapidly increasing and irreducible exophthalmos. Visual acuity could be severely decreased or even lost. In our series, 5 patients presented a negative light perception on admission.

Sinusitis is involved in at least two-thirds of orbital cellulitis in adults and $90 \%$ of cellulitis in children [1-9]. The second infectious origin described in the literature is skin infections and eye trauma with contaminated wound. In our study, the infectious origins are dominated by sinusitis, especially pansinusitis, which highlights the influence of delayed diagnosis until the spread of infection to other sinuses. Dacryocystitis predominated in adults followed by skin and oral infections [10]. Orbital cellulitis before the age of 5 years is almost always due to ethmoid sinusitis and the germs involved are Hemophilus influenzea and staphylococcus [11]. In our study, the most common germ was streptococcus, focusing on the severity of neighbouring infections (ENT sphere). However, many microbiological tests returned non-contributory and negative as a result of inappropriate initial treatment and self-medication.

Orbital CT scan is the imaging modality of choice in orbital cellulitis. It allows good visualization of orbital contents, sinus cavities, the surrounding structures and makes it possible to determine the stage of the cellulitis [1,9-12]. All of our patients received an urgent orbital CT scan confirming the diagnosis.

Once the diagnosis of orbital cellulitis is confirmed, the hospitalization with the institution of probabilistic broad-spectrum parenteral antibiotherapy is indicated without waiting for the paraclinical investigations results. The aim of treatment is to avoid the abscesses formation and to prevent their rupture and their spread to neighbouring structures. In this study, streptococcus was the main germ found, so we associated amoxicillin/clavulanic acid or ceftriaxone with aminoglycoside, metronidazole was added if there was any doubt about the presence of anaerobic germs. The addition of sys- temic corticotherapy helped relieve inflammation, improve antibiotics spread, and decrease pressure in the orbit in order to protect the optic nerve. Antibiotics and lubricating eye drops protected the cornea from ulceration and superinfection. Subperiosteal abscesses can be treated with parenteral antibiotics alone, especially in young patients, if the collection is $<10 \mathrm{~mm}$ with no mass effect on the medial rectus and in the absence of air bubbles suggesting an anaerobic infection [13]. In all other cases, particularly in the presence of severity signs such as a significant decrease in visual acuity, an afferent pupillary deficit (reflecting optic neuropathy), an ophthalmoplegia, a diplopia, an elderly patient, a collected orbital abscess or a clinical aggravation under medical treatment, surgery is recommended [1]. In the case of an accessible abscess, it can be evacuated by puncture drainage. However, if the abscess is deep, the surgical drainage guided by imagery is ensured by orbitotomy or by endonasal surgical technique.

The evolution of orbital cellulitis is unpredictable. In some cases, the eyeball can be spared for a long time, while in other cases, despite appropriate treatment, visual function may be threatened and lead to blindness [14]. This blindness can be secondary to an optic neuropathy of mechanical origin by intraorbital pressure elevation, or of vascular origin by ischemia, central retinal artery occlusion or thrombophlebitis, or of inflammatory origin (infectious neuritis). Retinal and choroidal vascular occlusions, retinal detachments and phthisis of the globe have also been described. Finally, a poor functional result can be linked to exposure keratitis or more exceptionally to retinal haemorrhages and retinal exudates [15].

\section{CONCLUSION}

Orbital cellulitis is a serious infection that can affect the visual prognosis, especially when the diagnosis is late and the treatment is inadequate. Sinus infections are mainly involved in this condition. Faced with the significant rate of functional complications, the ophthalmologist must make a rapid diagnosis, assess the ocular repercussions and initiate urgent medical treatment. Surgery will remain reserved for complications.

\section{Conflict of interest}

The authors do not declare any conflict of interest. 


\section{REFERENCES}

1. Mouriaux F, Rysanek B, Babin E, et al. Les cellulites orbitaires. J Fr Ophtalmol. 2012; 35(1): 52-57, doi: 10.1016/j.jfo.2011.08.004.

2. Wane $A M, B a E A$, Ndoye-Roth $P A$, et al. Une expérience sénégalaise des cellulites orbitaires. J Fr Ophtalmol. 2005; 28(10): 1089-1094, doi: 10.1016/s0181-5512(05)81143-x.

3. Kaimbo DK, Kiangalanga J, Missotten L. L'étude des exophtalmies au Zaire. Bull Soc Belge Ophtalmol. 1995; 259: 199-204.

4. Fanella $S$, Singer A, Embree J. Presentation and management of pediatric orbital cellulitis. Can J Infect Dis Med Microbiol. 2011; 22(3): 97-100, doi: 10.1155/2011/626809, indexed in Pubmed: 22942886.

5. Boivin L, Adenis JP. Infections orbitaires de l'enfant : clinique, imagerie et traitement. J Fr Ophtalmol. 2009; 32(5): 368-373, doi: 10.1016/j. jfo.2008.10.005.

6. Basraoui D, Elhajjami A, Jalal H. [Imaging of orbital cellulitis in children: about 56 cases]. Pan Afr Med J. 2018; 30: 94, doi: 10.11604/ pamj.2018.30.94.14672, indexed in Pubmed: 30344878.

7. Aidan $P$, Francois $M$, Prunel $M$, et al. Cellulites de la région orbitaire chez l'enfant. Arch Pédiatr. 1994; 1: 879-885.

8. Chandler JR, Langenbrunner DJ, Stevens ER. The pathogenesis of orbital complications in acute sinusitis. Laryngoscope. 1970; 80(9):
1414-1428, doi: 10.1288/00005537-197009000-00007, indexed in Pubmed: 5470225.

9. Seongmu L, Michael TY. Management of preseptal and orbital cellulitis. Saudi J Ophthalmol. 2011; 25: 21-29.

10. Kikkawa DO, Heinz GW, Martin RT, et al. Orbital cellulitis and abscess secondary to dacryocystitis. Arch Ophthalmol. 2002; 120(8): 1096-1099, indexed in Pubmed: 12149070.

11. Tzili N, Chefchaouni MC, El kh, et al. Cellulite rétroséptale de l'orbite chez I'enfant: à propos de 25 cas. Opht J Soc Maroc Ophtalmol. ; 2014(23).

12. Bouziane M, Saoud S, Benna N, et al. Apport de la TDM dans la cellulite orbitaire. JFR EPOS. ; 2014.

13. Ryan JT, Preciado DA, Bauman N, et al. Management of pediatric orbital cellulitis in patients with radiographic findings of subperiosteal abscess. Otolaryngol Head Neck Surg. 2009; 140(6): 907-911, doi: 10.1016/j.otohns.2009.02.014, indexed in Pubmed: 19467413.

14. Smelt GJ, Migdal CS. Acute blinding sinusitis. Br Med J (Clin Res Ed). 1983; 287(6398): 1051-1052, doi: 10.1136/bmj.287.6398.1051, indexed in Pubmed: 6412944.

15. Chaudhry IA, Shamsi FA, Elzaridi E, et al. Outcome of treated orbital cellulitis in a tertiary eye care center in the middle East. Ophthalmology. 2007; 114(2): 345-354, doi: 10.1016/j.ophtha.2006.07.059, indexed in Pubmed: 17270683. 